\title{
At-a-glance
}

\section{The Canadian Bikeway Comfort and Safety (Can-BICS) Classification System: a common naming convention for cycling infrastructure}

\author{
Meghan Winters, PhD (1); Moreno Zanotto, MSc (1); Gregory Butler, MSc (2)
}

Tweet this article

\begin{abstract}
There is no standard naming convention for cycling infrastructure across cities. Our aim was to develop a common nomenclature for cycling infrastructure in Canada, relevant to the context of public health practice. We drew on transportation engineering design guides and public health guidance to develop a bicycle facility classification system: the Canadian Bikeway Comfort and Safety (Can-BICS) classification system, a three-tiered classification scheme that groups five bicycle facilities based on safety performance and user comfort. Adopting consistent nomenclature as per the Can-BICS system will support regional and national surveillance efforts in public health, planning and sustainability.
\end{abstract}

Keywords: open data, active transportation, cycling, infrastructure, nomenclature

\section{Introduction}

Getting more people to cycle, more often, is a goal common to public health, sustainability and transportation agendas. ${ }^{1-4}$ Many cities assemble data on their cycling infrastructure and increasingly make these data publicly available through open data initiatives; however, there is no standard naming convention to describe cycling infrastructure. This lack of common nomenclature hinders research and practice efforts to understand the role of cycling infrastructure in supporting active travel across communities.

Our aim was to develop a common nomenclature for cycling infrastructure in Canada, relevant to the context of public health. Such nomenclature is a foundational step toward the operationalization of metrics that may be used for public health research and surveillance of physical activity in Canada. ${ }^{5}$ We considered cycling infrastructure to be part of roadways or paths intended for cycling (also referred to as "bicycle facilities" or "bikeways"); we did not include end-of-trip facilities such as cycle parking, lockers or showers, which are not consistently tracked.

This study is exempt from Research Ethics Board review as the research uses exclusively publicly available information for which there is no reasonable expectation of privacy.

\section{Methods}

\section{Overview}

We reviewed transportation engineering design guides and used public health guidance to develop a classification system based on safety performance (injury or crash risk along different infrastructure types) and user comfort (preferences for infrastructure types in terms of comfort

\section{Highlights}

- A common nomenclature for cycling infrastructure in Canada is needed to further public health surveillance efforts on active-transportation environments.

- The Can-BICS system is a threetiered cycling infrastructure classification system that reflects the safety performance and user comfort of five bicycle facility types.

- High-comfort bikeways are lowstress routes. These bikeways include cycle tracks on major streets, local street bikeways and cycle-only offstreet paths.

- Medium-comfort bikeways are lowto-medium stress routes. These bikeways include multi-use paths sited next to a roadway or along independent corridors.

- Low-comfort bikeways are highstress routes. These bikeways include painted bike lanes along busy roadways.

and stress). We also compiled cycling infrastructure names used in open data from Canadian municipalities and mapped them onto the nomenclature classification system.

\section{Engineering design guide review}

We reviewed national transportation engineering design guides from Canada and the USA published within the last 5 years

\section{Author references:}

1. Faculty of Health Sciences, Simon Fraser University, Burnaby, British Columbia, Canada

2. Public Health Agency of Canada, Ottawa, Ontario, Canada

Correspondence: Meghan Winters, Faculty of Health Sciences, Simon Fraser University, 8888 University Drive, Burnaby, BC V5A 1S6; Tel: 778-782-9325; Fax: 778-782-5927; Email: mwinters@sfu.ca 
to identify how cycling infrastructure types are defined and categorized. From these we identified other relevant documents. The documents reviewed were as follows: the Transportation Association of Canada (TAC) Geometric Design Guide for Canadian Roads ${ }^{6}$; the City of Vancouver Transportation Design Guidelines: All Ages and Abilities Cycling Routes ${ }^{7}$; CROW Design Manual for Bicycle Traffic ${ }^{8}$; NACTO's Urban Bikeway Design Guide and Designing for All Ages \& Abilities: Contextual Guidance for High-Comfort Bicycle Facilities ${ }^{10}$; and the Massachusetts Department of Transportation Separated Bike Lane Planning \& Design Guide ${ }^{11}$.

\section{Developing classification}

We analyzed the classification approaches and justification within each of the engineering guidelines to inform our proposed nomenclature. We also reviewed the public health literature on safety and preference for cycling infrastructure types because safety performance and user comfort were primary organizing principles for the classification scheme (for more information, see the review in The Canadian Bikeway Comfort and Safety (Can-BICS) Classification System: A Proposal for Developing Common Naming Conventions for Cycling Infrastructure report ${ }^{12}$ ). Our preliminary classification scheme was reviewed by one US and three Canadian experts in the fields of public health $(\mathrm{n}=2)$ and transportation planning and design $(n=2)$. Their feedback resulted in refined infrastructure definitions but no substantial changes to the classification.

\section{Open data analysis}

Our aim was to characterize the range of cycling infrastructure names used by Canadian communities to understand the scope of the nomenclature and how this interfaced with the proposed Can-BICS scheme. To select a national sample, we chose the $10 \%$ most populated census subdivisions from each province and territory. Taken together, these 45 census subdivisions covered $50.4 \%$ of the Canadian population.

We searched for a cycling infrastructure dataset and supplemented open data with a municipal cycling map when necessary. We extracted all names used in the municipal data and categorized these to the Can-BICS classification scheme. First, where possible ( $\sim 60 \%$ of names), we categorized facility names to the five Can-BICS cycling facilities by name alone (e.g. bike lanes categorized as painted bike lane). To ensure rigour, we performed spot checks on $10 \%$ of these facility names by using Google Street View (an online street view imagery service) and QGIS (a geographic information system) to locate and identify infrastructure types; all matched. Where the name itself did not facilitate easy categorization ( $\sim 40 \%$ ), we relied on Google Street View and QGIS. The open data files and facility name data are available through the SFU RADAR repository (researchdata .sfu.ca).

\section{Results}

The six engineering design guidelines identified multiple cycling facilities. We grouped these into five consolidated categories ("painted bike lanes," "local street bikeways," "cycle tracks," "bike paths" and "multi-use paths") related to design, exclusivity for cyclists and proximity of cyclists to other road users. ${ }^{6,7}$ Some guides touched on safety (e.g. separation from motor vehicles), but there was little explicit consideration of user preference or comfort, especially for roadway cycling facilities.

Integrating practice guidelines and public health considerations to categorize infrastructure that would best encourage cycling and make cycling safer, we developed the Canadian Bikeway Comfort and Safety (Can-BICS) classification system. This three-tiered classification scheme groups five cycling facilities based on safety performance and user comfort (Table 1):

- High-comfort bikeways. These lowstress cycling facilities are comfortable for most people. Route types include cycle tracks alongside busy roads, local street bikeways and off-road bike paths.

- Medium-comfort bikeways. These lowto-medium stress cycling facilities are considered comfortable by some people. The off-road infrastructure multiuse path fits within this category. Multi-use paths are shared with pedestrians and other active modes and can be located along a road or in an independent corridor.

- Low-comfort bikeways. These cycling facilities are high stress and comfortable for few people. The infrastructure type within this category is a painted bike lane, where people are cycling in a painted lane along busy roadways.

\section{Comparing open data facility names with Can-BICS}

Of the 45 municipalities, $89 \%$ ( $n=40 / 45$ ) had an open data catalogue and $80 \%$ of these included a cycling infrastructure dataset $(n=32 / 40)$. Data sources were published between 2005 and 2019. We extracted 269 cycling infrastructure names from open data (range: 2-14 per census subdivision) after removing obvious pedestrian infrastructure (e.g. stairs and sidewalks), route fragments and decommissioned routes. About 100 unique names were in use, after taking into account related terms (e.g. bike lane and bicycle lane). We categorized $60 \%$ of the 269 names to the five Can-BICS cycling facilities by name alone (e.g. bike lanes categorized as painted bike lane). The remaining $40 \%$ ( $n=108)$ we assessed via Google Maps Street View (see The Canadian Bikeway Comfort and Safety (Can-BICS) Classification System: A Proposal for Developing Common Naming Conventions for Cycling Infrastructure). ${ }^{12}$

We compared municipal open data nomenclature and Can-BICS (Figure 1) to assess overlap. Note that the proportions reported here represent the frequency of use of this facility name across the open data files and not the proportional distance of an infrastructure type within the cycling network. We found that $23 \%$ of names in open data were high-comfort bikeways: $8 \%$ being cycle tracks, $12 \%$ local street bikeways and $3 \%$ bike paths. Overall, $24 \%$ were medium comfort (multi-use paths) and $28 \%$ were low comfort (painted bike lanes).

There were also facility names that arose in open data but did not fit the Can-BICS criteria as they are not considered suitable (i.e. safe or comfortable) for promoting cycling for people of all ages and abilities based on the current state of knowledge. Many were shared lanes, that is, sharrows in a car travel lane. There is no evidence that sharrows provide the benefit of safety, and the majority of people do not want to share a travel lane with motor vehicles. Others were gravel trails, namely multiuse trails surfaced in gravel, dirt or aggregate, including mountain bike trails, walking trails in parks or hiking dirt paths. The Transportation Association of Canada guidelines explicitly exclude gravel trails, with the rationale that these are accessible to a smaller range of bicycles and have unique design requirements. ${ }^{6}$ Finally, "mixed 
TABLE 1

The Canadian Bikeway Comfort and Safety (Can-BICS) Classification System

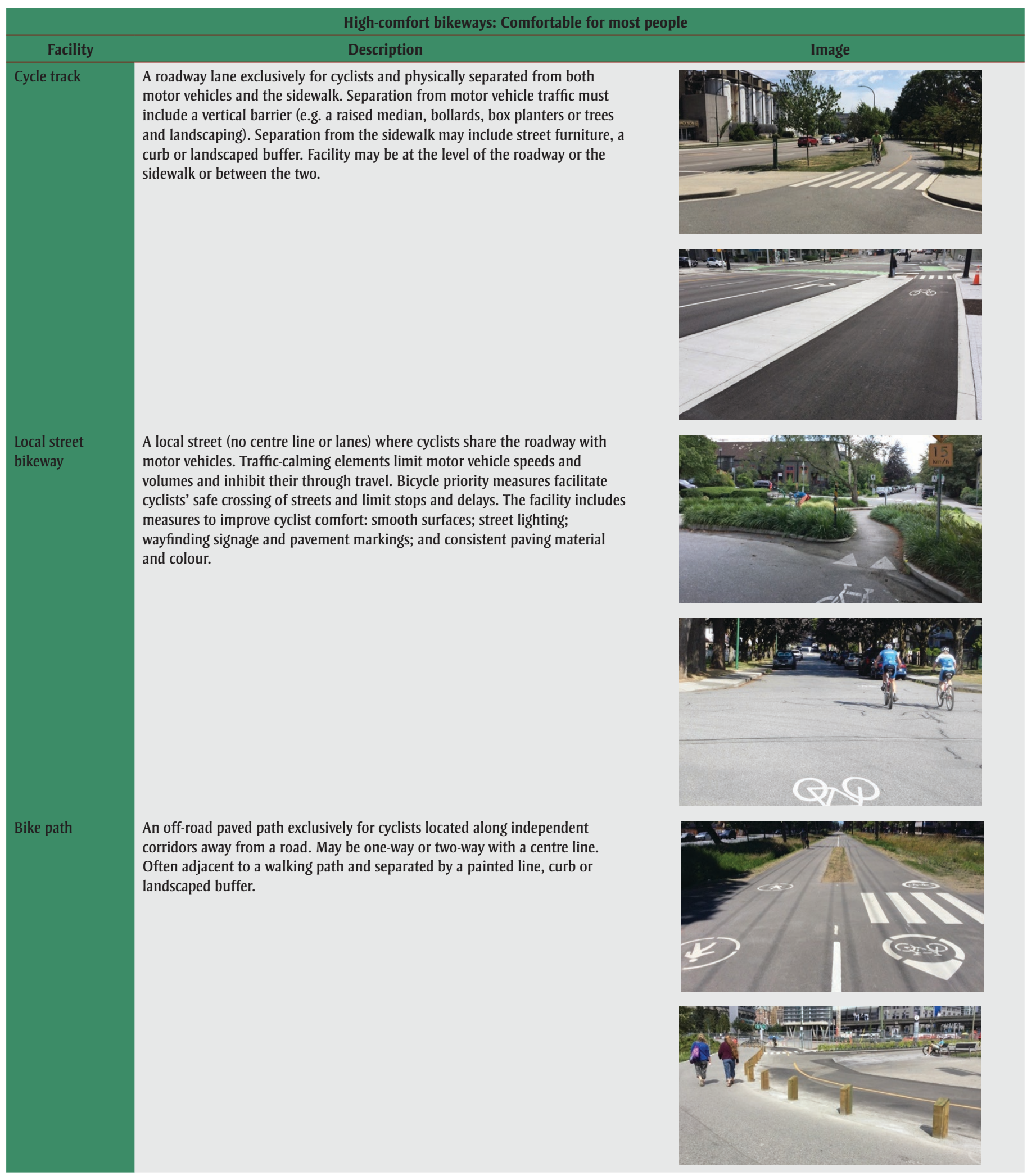

Continued on the following page 


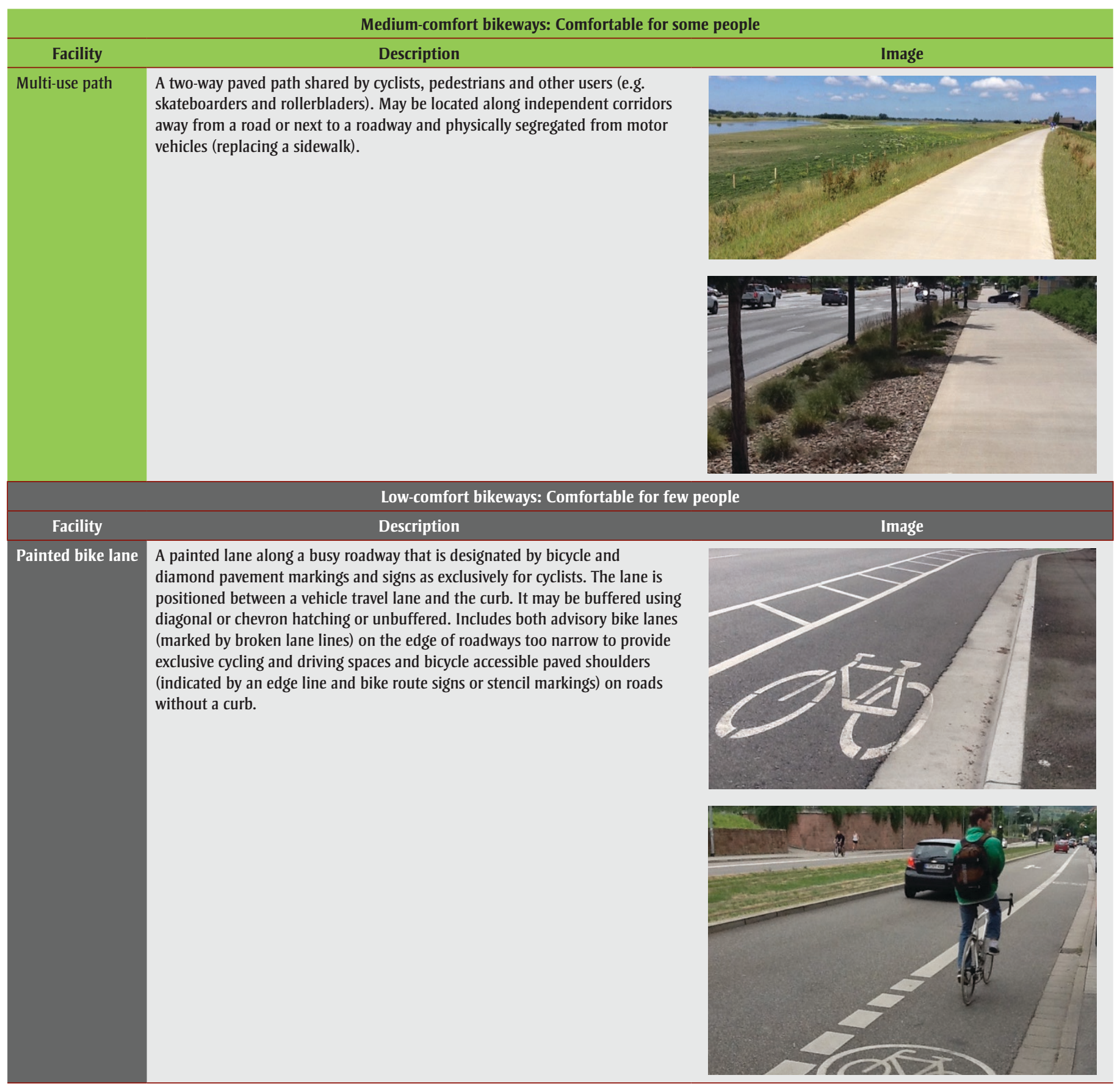

Source: The Canadian Bikeway Comfort and Safety (Can-BICS) Classification System: A Proposal for Developing Common Naming Conventions for Cycling Infrastructure. ${ }^{12}$

traffic" infrastructure (unimproved local roads) may serve as links to the main cycling network, but without signage or traffic calming, do not constitute cycling infrastructure. Together, these routes comprised $26 \%$ of the different facility names in open data.

\section{Discussion}

The Canadian Bikeway Comfort and Safety (Can-BICS) classification system defines five types of cycling facilities ordered into a three-tiered classification scheme based on safety performance and user comfort. The classification was informed by a review of professional practice guidelines for bicycle facility design, public health literature on safety and preferences and a scan of current naming conventions. This approach focuses on safer cycling facility types preferred by people of all ages and abilities, reflecting a public health perspective that aims to get more people cycling, more often, for both individual and population-level benefits. ${ }^{13}$

A standardized nomenclature approach for cycling infrastructure is essential for public health surveillance as it can enable comparisons of the availability and infrastructure types across settings and over time. We envision that planners can apply the standardized nomenclature in CanBICS to categorize the routes in their own communities and enable the development 
FIGURE 1

Categorization of municipal open data bicycle facility names ${ }^{\text {a }}$ to the Can-BICS classification system

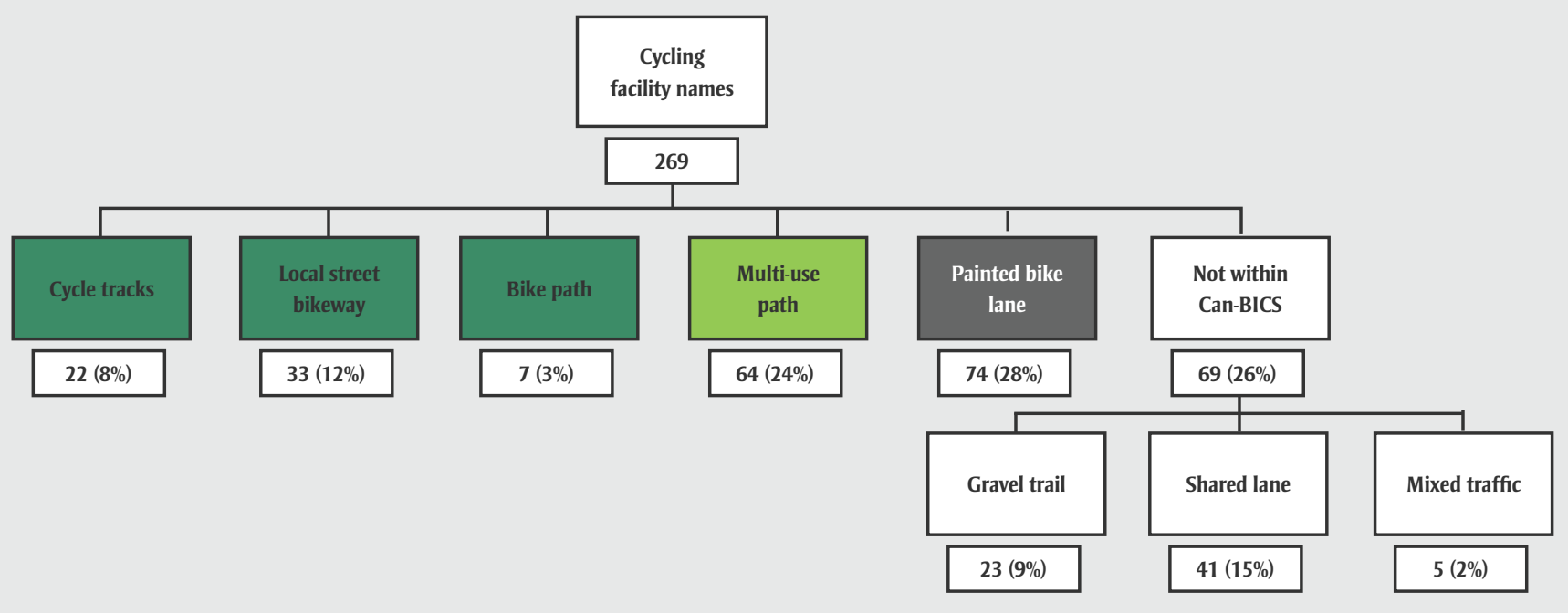

Source: The Canadian Bikeway Comfort and Safety (Can-BICS) Classification System: A Proposal for Developing Common Naming Conventions for Cycling Infrastructure. ${ }^{12}$

Abbreviation: Can-BICS, Canadian Bikeway Comfort and Safety.

269 facility names from across 45 census subdivisions.

of granular spatial data and metrics to support local public health authorities.

For Can-BICS, next steps are to operationalize metrics (e.g. kilometres of high/ medium/low-comfort routes per area), identify spatial units (e.g. dissemination area) and boundary issues, and evaluate the quality of open data sources. Emerging work suggests OpenStreetMap (OSM; openstreetmap.org) is a promising data source for Canadian cities. ${ }^{14}$ For a national effort, any data source must be evaluated in terms of access, completeness and comparability, but the potential to streamline and standardize efforts is strong.

\section{Strengths and limitations}

Design matters. While Can-BICS uses a broad classification of user comfort and safety, there are nuances. A cycle track (high comfort) that is poorly designed may have greater injury risk than a welldesigned painted bike lane (low comfort). Intersection treatments and network connectivity also impact route safety and comfort.

\section{Conclusion}

There is limited past work in harmonizing the names for cycling infrastructure across cities, although this is important for comparing neighbourhoods within a single city or a set of cities as part of a national approach. ${ }^{15,16}$ A standardized nomenclature such as Can-BICS is a foundational step toward building capacity in public health surveillance for urban cycling environments.

\section{Acknowledgements}

This study was funded by the Public Health Agency of Canada (Award \#4500387514). Meghan Winters is supported by a Scholar Award from the Michael Smith Foundation for Health Research.

We thank the city governments who made available open data. We also thank four public health and traffic engineering experts who provided feedback on earlier versions of the classification system and two individuals who provided French translation support.

\section{Authors' contributions and statement}

MW led the project conceptualization, supervised the analysis and contributed to the drafting and revision of the paper. MZ led the literature review, data collection and data analysis and contributed to the drafting and revision of paper. GB contributed to the project conceptualization and the drafting and revision of the paper

The content and views expressed in this article are those of the authors and do not necessarily reflect those of the Government of Canada.

\section{References}

1. Pucher J, Buehler R. City cycling. Cambridge (MA): MIT Press; 2012. doi:10.7551/mitpress/9434.001.0001.

2. Winters $M$, Teschke $K$, Grant $M$, Setton EM, Brauer M. How far out of the way will we travel? Transp Res Rec. 2010;2190(1):1-10. doi:10.3141 /2190-01.

3. Winters M, Teschke K. Route preferences among adults in the near market for bicycling: findings of the Cycling in Cities Study. Am J Health Promot. 2010;25(1):40-7. doi:10.4278 /ajhp.081006-QUAN-236.

4. Teschke K, Harris MA, Reynolds CC, et al. Route infrastructure and the risk of injuries to bicyclists: a case-crossover study. Am J Public Health. 2012;102(12):2336-43. doi: 10.2105/AJPH.2012.300762.

5. Roberts KC, Butler G, Branchard B, et al. The Physical Activity, Sedentary Behaviour and Sleep (PASS) Indicator Framework. Heal Promot Chronic Dis Prev Can. 2017;37(8):252-6. doi: 10.24095/hpcdp.37.8.04.

6. Transportation Association of Canada. Chapter 5 - Bicycle integrated design. In: Geometric design guide for Canadian roads. Ottawa (ON): Transportation Association of Canada; 2017. 
7. City of Vancouver. Transportation design guidelines: all ages and abilities cycling routes. Vancouver (BC): City of Vancouver; 2017. https://vancouver .ca/files/cov/design-guidelines-for -all-ages-and-abilities-cycling-routes .pdf

8. Platform CROW. Design manual for bicycle traffic. Ede (NL): CROW; 2016.

9. National Association of City Transportation Officials. Urban bikeway design guide, 2nd ed. Washington (DC): NACTO; 2014.

10. National Association of City Transportation Officials. Designing for all ages \& abilities: contextual guidance for high-comfort bicycle facilities. Washington (DC): NACTO; 2017 Dec. https://nacto.org/publication/urban -bikeway-design-guide/designing -ages-abilities-new/

11. Massachusetts Department of Transportation. Separated bike lane planning \& design guide [Internet]. Boston (MA): MassDOT; 2015 [cited 2017 Dec 4]. Available from: https://www .mass.gov/lists/separated-bike-lane -planning-design-guide

12. Winters M, Zanotto M. The Canadian Bikeway Comfort and Safety (CanBICS) Classification System: a proposal for developing common naming conventions for cycling infrastructure. Vancouver (BC): CHATR; 2019. Available at: https://chatrlab.ca /projects/

13. Schepers P, Twisk D, Fishman E, Fyhri A, Jensen A. The Dutch road to a high level of cycling safety. Saf Sci. 2017;92:264-73. doi:10.1016/j.ssci.2015 .06 .005 .

14. Ferster C, Fischer J, Manaugh K, Nelson $\mathrm{T}$, Winters M. Using OpenStreetMap to inventory bicycle infrastructure: a comparison with open data from cities. Int J Sustain Transp 2020; 14(1):64-73. doi:10.1080/15568318 .2018.1519746.

15. Winters $M$, Teschke $K$, Brauer $M$, Fuller D. Bike Score ${ }^{\circledR}$ : associations between urban bikeability and cycling behavior in 24 cities. Int J Behav Nutr Phys Act. 2016;13(1):18. doi:10.1186 /s12966-016-0339-0.
16. Vijayakumar N, Burda C; Pembina Institute. Cycle cities: supporting cycling in Canadian cities. Toronto (ON): Pembina Institute; [updated 2015 Dec 16]. 